\title{
Real-Time DC Servomotor Identification and Control of Mechanical Braking System for Vehicle to Vehicle Communication
}

\author{
Mohammad A. Obeidat, PhD \\ Tafila Technical University \\ Zip code 66110, P.O.Box 179, Tafila, Jordan
}

\begin{abstract}
Remotely-controlled DC Servomotors must transmit phase angle measurements and receive control commands via communication channels. Sampling, quantization, data transfer, and signal reconstruction are mandatory in such networked systems. Real Time Vehicle-To-Vehicle (V2V) communications system is designed to transfer information between vehicles, this information provides warnings to drivers and other vehicles. Transferring vital information between vehicles improves the overall efficiency and safety of the roadways. One of the vital information is braking data. This paper develops parameter estimation and system identification of a DC servomotor using quantized phase measurements. Then, through wireless communication channels braking data collecting from DC servomotor identification system is transferred between vehicles to help drivers avoid sudden braking accidents. The developed closed loop system with wireless communication channels in present of noise efficiently affect feedback performance. Simulations and experimental studies are performed to illustrate potential applications of this technology.
\end{abstract}

\section{Keywords}

DC servomotor, Identification, braking, Vehicle, Closed Loop, Vehicle to Vehicle Communication.

\section{INTRODUCTION}

In this paper a remotely controlled closed loop electrical braking system is designed and developed. In the developed system uses a Flexi force sensor to convert the force on the pedal of the mechanical brake to a voltage. The electrical voltage signal $u_{k}$ is processed through microcontroller to drive a servomotor at a certain phase angle $\theta_{\mathrm{k}}$. Angle $\theta_{\mathrm{k}}$ allows the fluid in the mechanical braking system to flow in the cylindrical pipe brake. Through communication channels between vehicles, $\theta_{\mathrm{k}}$ is sent to achieve excellent driving behavior to reduce accidents. To evaluate the performance of the system, a prototype has been implemented to generate simulation results. The achieved results show excellent performance of the addressed system.

Transportation makes human life easy in many fields, it saves time and makes human life moving faster. Real Time VehicleTo-Vehicle (V2V) communications system is designed to transfer information between vehicles, this information provides warnings to drivers and other vehicles. Transferring vital information between vehicles improves the overall efficiency and safety of the roadways. V2V communication technology can prevent automobile accidents before they occur. This paper investigates unique issues rising from feedback control of electric motors with embedded communication channels. Design variables for the communication systems such as sampling schemes and quantization levels, for signal estimation such as parameter updating step sizes, and controllers such as controller gains, interact and jointly affect feedback performance [1].

Many advantages for wireless V2V technologies such as: realtime traffic information, personalized roadside assistance, automobiles to monitor and adjust position on highways, alert drivers if they are drifting out of their lane and slowing down if they get too close to the car around them.

Programmable microcontrollers are embedded in vehicles to control the actions of the actuators such as: solenoids, LCD displays, relays, switches depending on data collected from sensors such as: humidity, temperature, light level, and distance, etc.

One of the main systems in vehicles is the braking system, so transferring braking data between vehicles through warnings to the drivers if something suddenly happened, can reduce damage, accidents, and hardship. Servomotor controlled system can be added to the mechanical braking system in vehicles to control brake strength by controlling the fluid flow to the braking pads. A DC Servomotors offer some appealing features such as: moving to a particular angle, position and velocity with high efficiency and great precision. Also, small size, phase angle range, and the ability to control torque. DC servomotors can be used in many applications that required pressing, holding, pushing and twisting motions at very precise torques $[2,3]$.

Mechanical brakes are assemblies consisting of mechanical elements for the slowing or stopping of shafts in equipment drives. They use levers or linkages to transmit force from one point to another. Braking slows or stops the movement of the coupled shafts. Brakes are required to stop the vehicle within the smallest possible distance by converting the kinetic energy into the heat energy which is dissipated into atmosphere.

There are four main types of brakes:1) hydraulic disc brakes 2) hydraulic drum brakes 3 ) power brakes such as: air brakes, air hydraulic, vacuum, and electric brakes 4) antilock brake system (ABS) [4].

\subsection{Hydraulic Drum Brakes}

A drum brake assembly consists of a cast drum that is bolted to and rotates with the wheel. Inside the drum, there is a backing plate that has a set of brake shoes attached to it. Other components are attached to the backing plate, including a hydraulic cylinder and several springs and linkages. Figure 1 below shows drum brakes. 


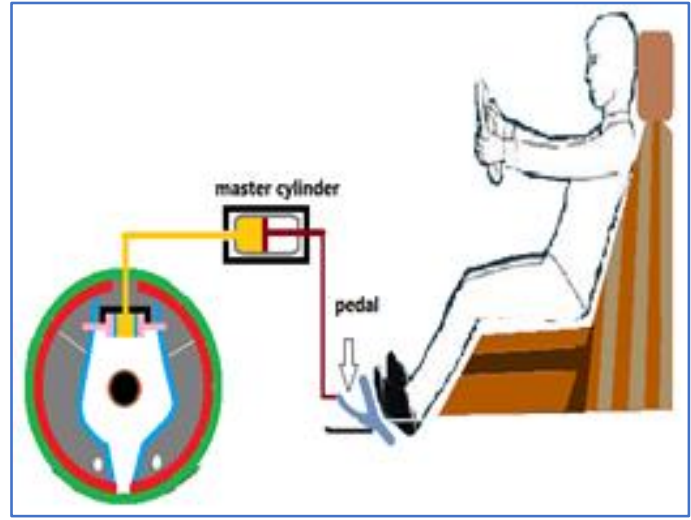

Fig 1: Drum brake system

\subsection{Hydraulic Disc Brakes}

Disc brakes are used on the front of the vehicle, while drum brakes are used on the rear wheels. Figure 2 shows disc brake system. The friction is produced by pads, as shown in Figure 2. These pads are squeezed or clamped against a rotating disc. The disc, also called the rotor, is attached to the rim and tire. The rotor is made of cast iron that is machined on both sides. The pads are attached to metal plates that are actuated by pistons from the hydraulic system.

In hydraulic brake, the automotive braking system uses hydraulic pressure to transfer the force of the operator's foot to press the friction surfaces together. When the foot pedal is pressed, a pressure is built up in the master cylinder. This pressure is then transferred throughout. the hydraulic lines to each wheel cylinder. Note that the pressure at each point in the system is the same.

Figure 3 shows the braking system for both pushing and releasing pedal operations.

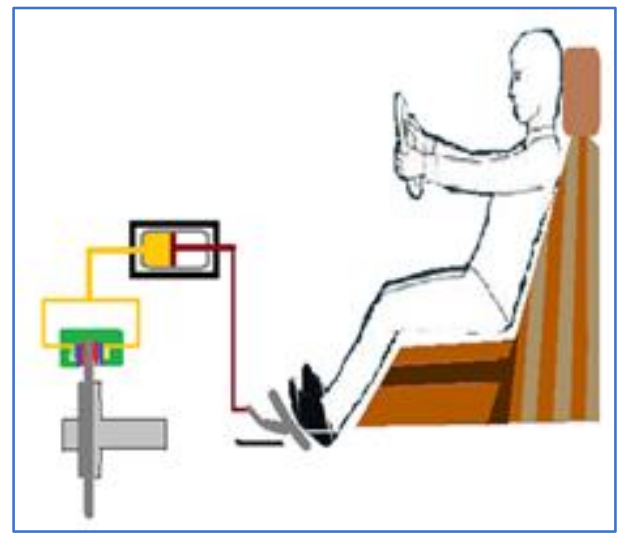

Fig 2: Disc brake system

\subsection{Power Brakes}

This type of brakes used engine or battery power to enhance braking system

Vacuum Brakes: Vacuum brake system is controlled through a brake pipe connecting a brake valve in the driver's cab with braking equipment on every vehicle. The operation of the brake equipment on each vehicle depends on the condition of a vacuum created in the pipe by an ejector or exhauster.

2. Air Brakes: The operation of air brakes is similar to hydraulic brake except that compressed air is used to apply brakes instead of hydraulic pressure. Air brakes are commonly used on heavy vehicles like trucks, buses.

\subsection{Antilock Brake System (ABS):}

It is used to avoid skidding. A modern ABS consists of an electronic control unit (ECU)., one sensor on each wheel, an electrically driven hydraulic pump and pressure accumulator is used to store hydraulic fluid to maintain high pressure in braking system. It is charged with nitrogen gas. ECU monitors and controls the antilock function when required [4,5].

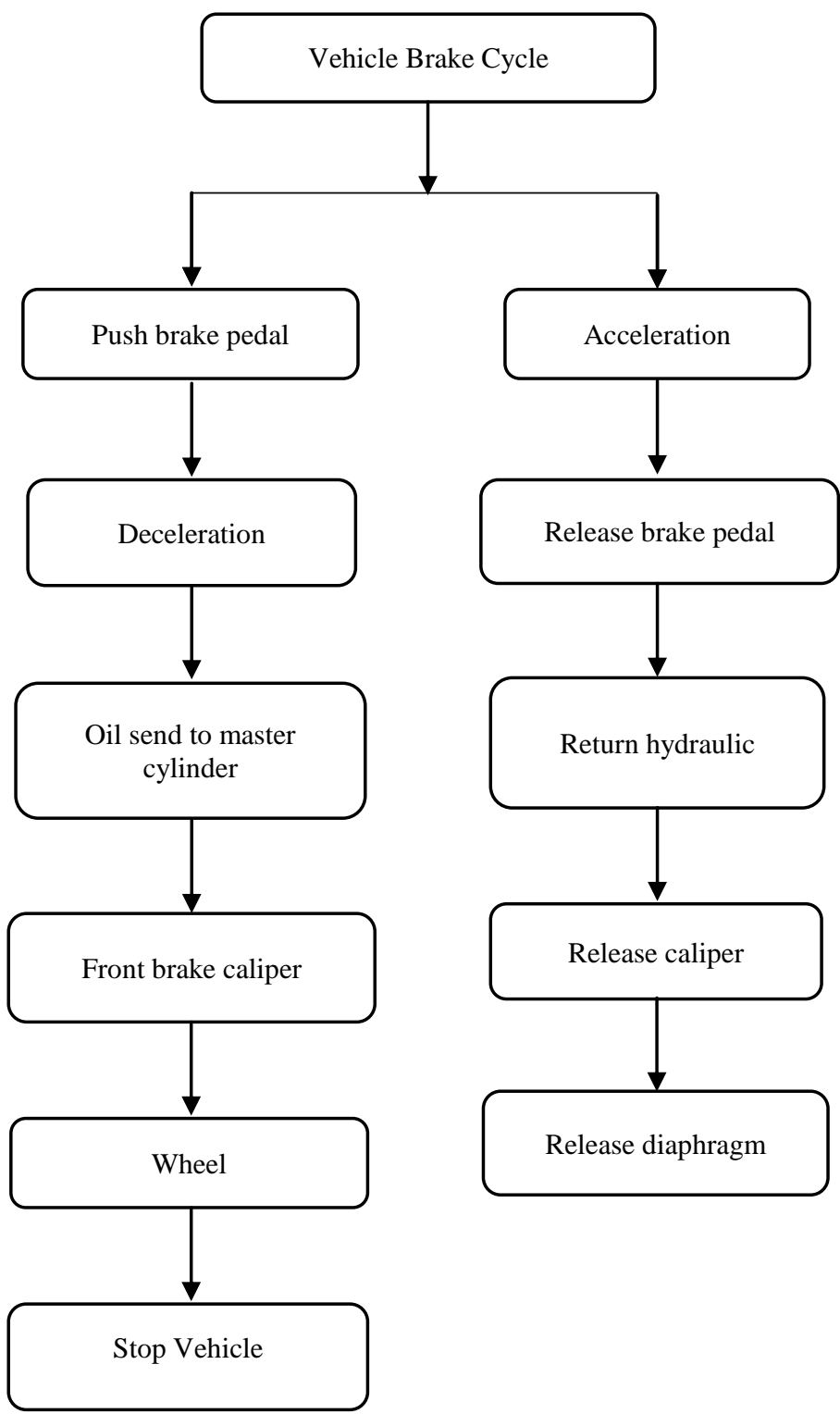

Fig 3: Braking system for both pushing and releasing pedal operations.

\section{MODELS OF DC SERVOMOTOR}

In this section the models for DC servomotor is derived. Starting from time domain differential equations, state space representation using Laplace transformation, which converted to discrete form using Z-transformation, and finally auto regression moving average models (ARMA) is proposed to identify the DC servomotor. After identifying the DC servomotor transfer function in ARMA model, then the parameters of the servomotor can be estimated. Real time data can be transferred between vehicles to control braking system in vehicles network. The DC servomotor system can be added to the vehicle as shown in Figure 4. 


\section{wireless data to be} transferred throush communication channel

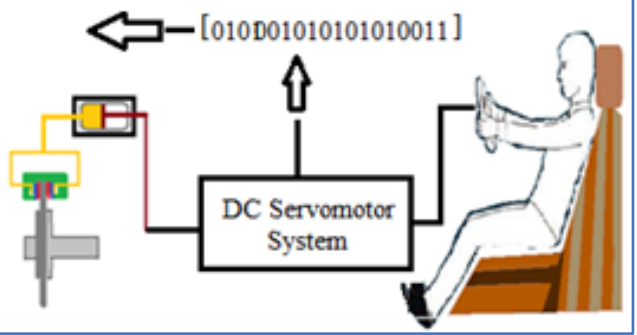

Fig 4: Modified Disk braking using DC servomotor system

The equations for the motor rotor and shaft motion

and stator wiring are:

$$
\begin{aligned}
& \frac{d \omega(t)}{d t}=\frac{-\mu}{J} \omega(t)+\frac{k_{m}}{J} i_{a}(t)-\frac{1}{J} T_{L}(t), \\
& \frac{d i_{a}(t)}{d t}=\frac{-k_{b}}{L_{a}} \omega(t)-\frac{R_{a}}{L_{a}} i_{a}(t)+\frac{1}{L_{a}} U(t),
\end{aligned}
$$$$
\frac{d \theta(t)}{d t}=\omega(t), \theta(s)=\frac{1}{s} \Omega(s)
$$

State space is: $\quad \dot{x}(t)=A_{s} x(t)+B_{s} u(t)$, and $\quad y(t)=$ $C_{s} x(t)$, Where

$$
\begin{array}{r}
A_{s}=\left[\begin{array}{cc}
-\mu / J & k_{m} / J \\
-k_{b} / L_{a} & -R_{a} / L_{a}
\end{array}\right] \\
C_{s}=\left[\begin{array}{ll}
1 & 0 \\
0 & 1
\end{array}\right], B_{s}=\left[\begin{array}{cc}
-1 / J & 0 \\
0 & 1 / L_{a}
\end{array}\right] \\
G(s)=C_{S}\left(S I-A_{s}\right)^{-1} B_{S}
\end{array}
$$

In particular, the transfer function from the input voltage $U(s)$ to the phase angle $\Theta(s)$ is

$G_{1}(s)=\frac{\Theta}{U}=\frac{\frac{k_{m}}{J} \frac{1}{L_{a}}}{s\left(\left(s+\frac{\mu}{J}\right)\left(s+\frac{R_{a}}{L_{a}}\right)+\frac{k_{m}}{J} \frac{k_{b}}{L_{a}}\right)}$

Denote $r r=\frac{R_{a}}{L_{a}}+\frac{\mu}{J}, d d=\frac{R_{a} \mu+k_{m} k_{b}}{L_{a} J}, g=\frac{k_{m}}{L_{a} J}$. It follows that $G_{1}(s)=\frac{g g}{s\left(s^{2}+r s+d\right)}=\frac{g}{s\left(s-s_{1}\right)\left(s-s_{2}\right)} \quad$ where $s_{1}=$ $\left(-r r+\sqrt{r r^{2}-4 d d}\right) / 2, \quad s_{2}=\left(-r r-\sqrt{r r^{2}-4 d d}\right) / 2$, and $\mathrm{s} 3=0$. are the poles of $G_{1}(s)$.

\subsection{Discretization of DC Servomotor Models}

For system identification, it is convenient to use a discretized model in a regression structure. Suppose that the sampling interval is $T$ (second). Denote the sampled signals

$$
\theta_{k}=\theta(k T), u_{k}=U(k T), k=0,1, \ldots
$$

Using the partial fraction expansion and zero-order hold function, the corresponding discrete-time transfer function of the sampled system can be derived via the standard $\mathrm{z}-$ transform as

$\frac{\widetilde{\theta}(z)}{\widetilde{U}(z)}=z\left(\frac{G_{1}(s)\left(1-e^{-T s}\right)}{s}\right):=\widetilde{G}_{1}(z)$.
Here, $\widetilde{\Theta}(z)$ and $\widetilde{U}(z)$ are the z-transforms of the speed and voltage sampled sequences, respectively.

Under a step input $\mathrm{v}_{\mathrm{S}}$ with amplitude $\mathrm{A}_{\mathrm{M}}$, the angular speed is $\widetilde{\Theta}(z)=\tilde{G}_{1}(z)\left(\frac{A_{M}}{1-z^{-1}}\right)$. This implies that

$\theta_{k}=A_{M}\left(c_{1}+c_{2} e^{s_{1} k_{T}}+c_{3} e^{s_{2} k_{T}}\right)$,

Where $c_{1}=\frac{p}{s_{1} s_{2}}, c_{2}=\frac{p}{\left(s_{1}\left(s_{1}-s_{2}\right)\right)}, c_{3}=\frac{p}{\left(s_{2}\left(s_{2}-s_{1}\right)\right)}$.

\subsection{Regression Models}

For system identification experiments. it is desirable to transform a model into a regression form. The general form for the speed can be written in an autoregressive moving average with input (ARMA or ARMAX) ${ }^{1}$ model [7-9] as

$$
\theta_{k}=\sum_{j=1}^{n} a_{j} \theta_{k}-j+\sum_{i=0}^{n} b_{i} u_{k}-i,
$$

Where $n$ is the model order. In our case, the PMDC motor is assumed to have the

following ARMA model $(n=2)$ structure

$\theta_{k}=\left[\theta_{k-1}, \theta_{k-2}, u_{k-1}, u_{k-2}\right]\left[\begin{array}{l}a_{1} \\ a_{2} \\ b_{1} \\ b_{2}\end{array}\right]$

Where $\theta_{k}$ is the angle of DC servomotor ( $\mathrm{rad}$ ) and $u_{k}$ the input voltage of DC servomotor $(V)$. It is a standard but tedious process to verify that the parameters are related to the original system parameters and the sampling interval by

Now the noise-free speed can be written in an operator form as

$$
\theta_{k}=\tilde{G}(q) u_{k}=\frac{b_{1} q+b_{2} q^{2}}{1-\left(a_{1} q+a_{2} q^{2}\right)} u_{k}, k=0,1,2, \ldots
$$

Where $\mathrm{q}$ is the one-step shift operator $\theta_{k}=\emptyset_{k}^{T} h, k=$ $0,1,2, \ldots$

Where $\emptyset_{k}^{T}=\left[\theta_{k}-1, \theta_{k}-2, u_{k}-1, u_{k}-2\right]$,

$$
h_{r}=\left[a_{1}, a_{2}, a_{3}, b_{1}, b_{2}, b_{3}\right] \text {. }
$$

The parameter vector $\mathrm{hr}$ is to be identified. When a random noise or dither $d_{k}$ is added to the output $\theta_{k}$, where

$$
y_{k}=\theta_{k}+d_{k} .
$$

Explaining the methodology of signal estimation which was introduced in [6-8] and some essential derivation steps that will be relevant in our study. The output angle signal $\theta_{k}$ is used in describing the algorithms and main features. The estimation steps and features for the control signal uk will be similar. The true heart rate $\theta_{k}$ is bounde $\theta_{\min } \leq \theta_{k} \leq \theta_{\max }$. $\theta_{k}$ is either measured with a measurement noise or added with a random dither $d_{k}$ to enhance signal estimation.

The noise-added signal $\theta_{k}+\mathrm{d}_{\mathrm{k}}$. is quantized to produce a quantization sequence $S\left(\theta_{k}+\mathrm{dk}\right)$, where $S$ represents the quantization function. More precisely, suppose that the signal $\theta_{k}+\mathrm{dk}$ is quantized by $\mathrm{m}$ quantization thresholds $\{\mathrm{h} 1, \ldots$, $\mathrm{hm}$, which divides the range [ $\theta \min , \theta \max ]$ into $\theta \min <\mathrm{h} 1<$. . $<\mathrm{hm}<\theta \max$. The output of the quantizer takes $\mathrm{m}+1$ possible values $\{1,2, \ldots, \mathrm{m}+1\}$ and is represented by:

$s_{k}=\sum_{i}^{m+1} i I\left[h_{i-1}<\theta_{k}+d_{k} \leq h_{i}\right.$. 
with $\mathrm{h} 0=0$ and 1 being the indicator function. In the special case of a binary-valued quantization of threshold $h$,

$$
s_{k}=\left\{\begin{array}{l}
1, \theta_{k}+d_{k} \leq h \\
0, \theta_{k}+d_{k}>h
\end{array}\right.
$$

For clarity, the binary-valued quantization is used to derive algorithms and properties. Generalization to $\mathrm{m}$ quantizing levels can be found in [7-11]]. sk will be processed to estimate $\theta_{k}$ at the receiver side.

\subsection{Signal Estimation Algorithms}

For a selected $0<\alpha<1$, define the following truncated and exponentially weighted empirical measures:

$\lambda_{k}^{\Delta}=(1-\alpha) \sum_{l=-\infty}^{k} \alpha^{k-1} s_{i} \ldots$

where the weight is normalized so that when

$$
s_{l}=1,(1-\alpha) \sum_{l=0}^{\infty} \alpha^{l}=1
$$

This algorithm can also be written recursively as

$$
\begin{aligned}
\lambda_{k}^{\Delta}= & \lambda_{k-1}^{\Delta}+(1-\alpha)\left(s_{k}-\lambda_{k-1}^{\Delta}\right) \\
& =\lambda_{k-1}^{\Delta}+\beta\left(s_{k}-\lambda_{k-1}^{\Delta}\right)
\end{aligned}
$$

which is a stochastic approximation algorithm with a constant step size $\beta=1-\alpha$. To understand the meaning of the weight $\alpha$ and the step size $\beta=1-\alpha$, note that (2) is a weighted averaging computation. The smaller the $\alpha$ value, the faster the decaying rate $\alpha \mathrm{k}-1$ in (2), which in turn implies the averaging uses mostly the recent data, that is a small data window in the signal averaging. This is equivalent to $\beta$ being close to 1 . This represents a fast updating algorithm. Such an algorithm will be able to track fast changing signals, but will have less capability in attenuating noise effects. However, this is a fast response filter (i.e., less dynamic delay) and hence will have less detrimental effects on feedback stability and performance. This intuitive understanding will help in interpreting case study results. In addition, when the step sizes is translated to the actual time, each updating step in signal estimation means Ts second. Consequently, the sampling period is a fundamental parameter when feedback performance is evaluated. For a technical delicacy, for some small $\delta$ satisfying $0<\delta<1$, define:

$$
\lambda_{k}=\left\{\begin{array}{cc}
\lambda_{k}^{\Delta}, & \delta<\lambda_{k}^{\Delta}<1-\delta \\
\delta, & \lambda_{k}^{\Delta}<\delta \\
1-\delta, & \lambda_{k}^{\Delta}>1-\delta,
\end{array}\right\} \ldots \ldots \ldots(3)
$$

Example 1 Suppose that a DC Servomotor has the following parameters (from the manufacturer): $\mathrm{La}=2.75 \mathrm{E}-6 \mathrm{H}, \mathrm{Ra}=4.0$ ohm, J = 3.2284E-6Kg.m2, $\mu=3.5077 \mathrm{E}-6$ N.m.s, $\mathrm{K}=0.0274$ N.m/A or V/rad/s. The system is sampled with the sampling period Ts $=0.01$ second. In an open-loop environment, Figure 5 shows the step responses (when PI controller is applied) of the original continuous-time model and its discrete system. It is clear that the sampling interval is adequate for the sampled system to approximate the original continuous-time system.

To further demonstration of performance, Figures 6 and 7 present the ramp and sinusoidal responses of the closed-loop system with the PI controller. In all above cases, it can be observed that without communication links, the controller performs well in terms of stability and performance. Also, the discretized models are sufficiently accurate as approximations for system identification and control, indicating that the sampling interval $\mathrm{Ts}=0.01$ is suitable.
This will not affect system analysis. Then, the estimation of vk is:

$\hat{\theta}_{k}=h-F^{-1}\left(\lambda_{k}\right) \ldots \ldots \ldots$

Figures 5, 6, and 7 show the step, ramp, and sinusoidal responses of the closed loop DC servomotor for continuous and discrete. Figure 6 shows the developed DC servomotor identification closed loop system with communication channels between vehicles. From the figure it can be seen that the signal is transmitted through binary sensor as binary sequence, then the received binary sequence will be estimated using algorithm explained in equations (1-4). The estimated signal will be matched with the transmitted signal through error correction that will excite the PI controller to achieve the optimal angle of pressed hydraulic flow to the braking pads.

\subsection{Servomotor Feedback Systems with Communication Channels}

It can be shown that adding the signal estimation algorithm (4) into the DC Servomotor feedback loop system can be represented by a signal averaging filter and an equivalent noise source. Consequently, the block diagram of the closed loop DC servomotor is expanded with one filter $\mathrm{Fb}$ such that [12-17]

$$
F_{b}=\frac{(1-\alpha) z}{z-\alpha}
$$

Fig. 8 shows the overall closed loop DC servomotor system

When a DC Servomotor must be remotely controlled, communication channels are inserted into the feedback loop, leading to a new simplified closed loop system shown in Figure 9 and signal estimation process in Figure 8 . The overall system consists of the DC Servomotor transfer function $\breve{G}(\mathrm{z})$, the PI controller GC(z), communication blocks in both output and input sides. In this configuration, the output phase angle signal $\theta(t)$ and the control signal uk are communicated through communication channels, and then estimated. In our development, the two communication blocks will be used and signal estimation algorithms to have different sampling intervals and step sizes, in order to accommodate realistic wireless communication networks. Inserting a communication block to transmit a signal in the feedback loop introduces some errors; and signal estimation leads to dynamic delays. The aim of this paper is to study the behavior of the closed-loop system under the communication channels and signal estimation algorithms. 


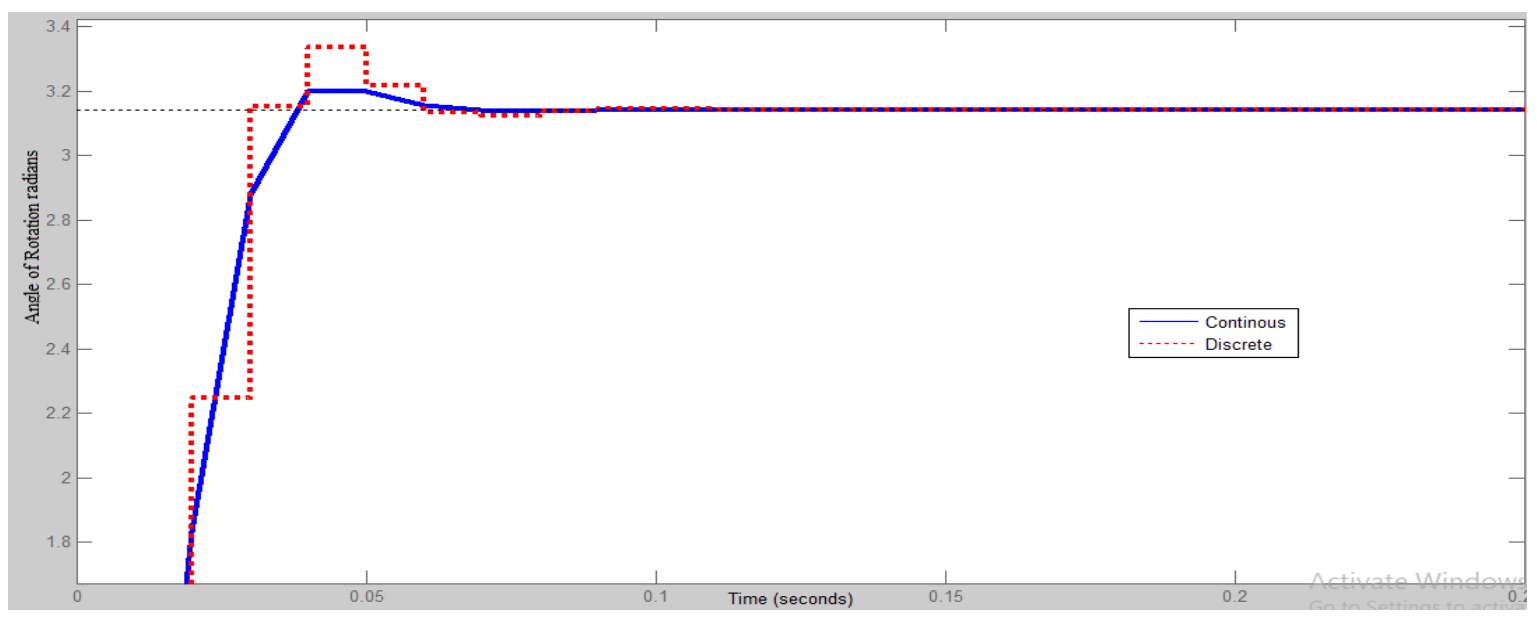

Fig 5: Continuous and discrete step responses of DC servomotor

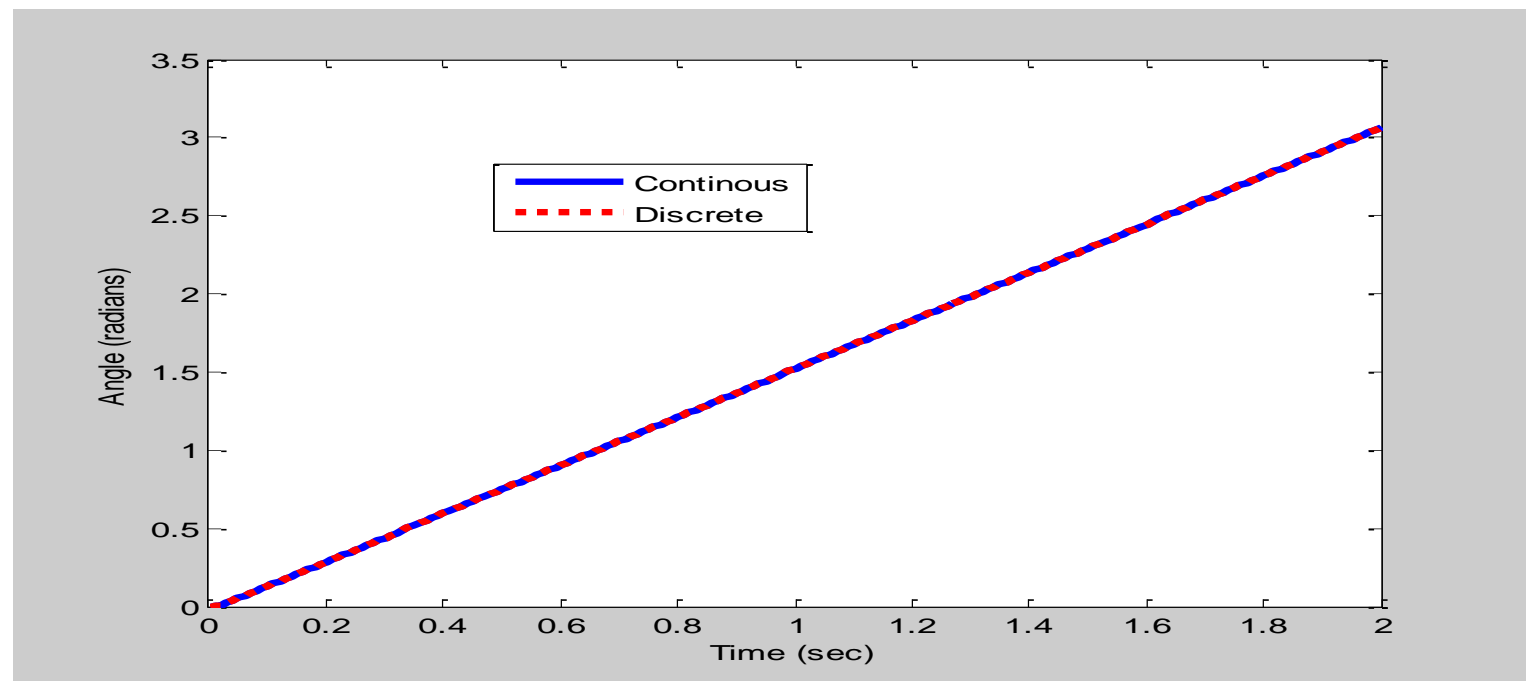

Fig 6: Continuous and discrete ramp responses of DC servomotor

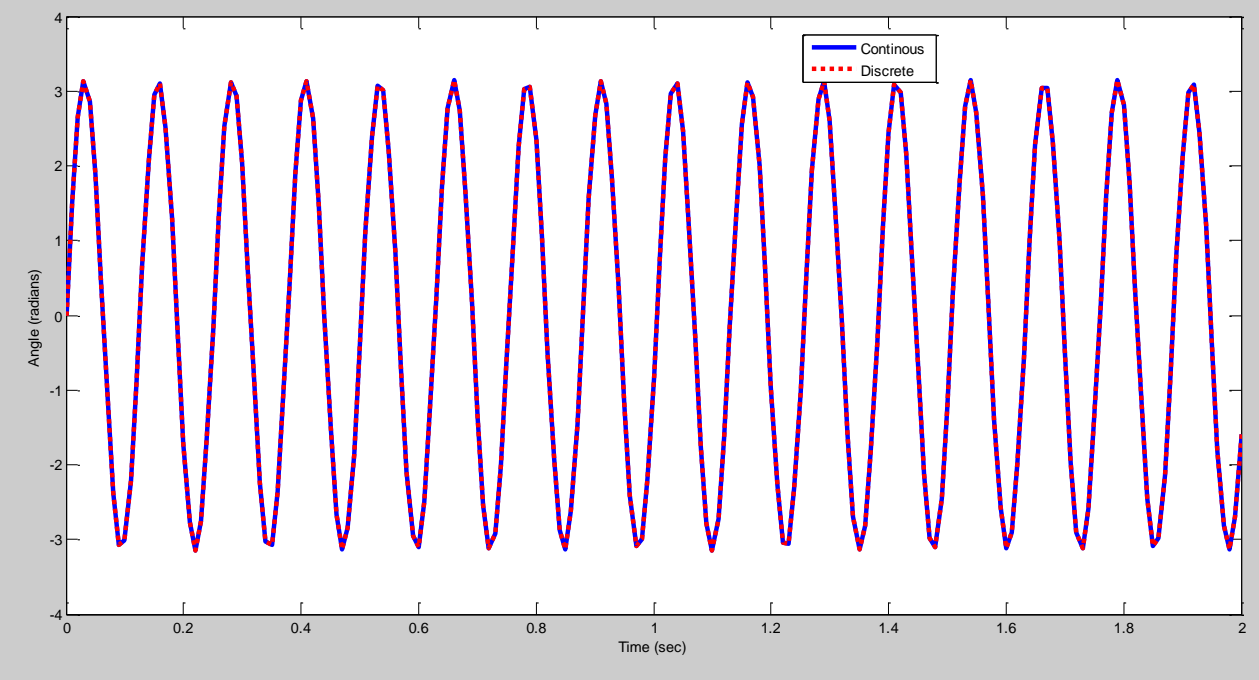

Fig 7: Continuous and discrete sinusoidal responses of DC servomotor 


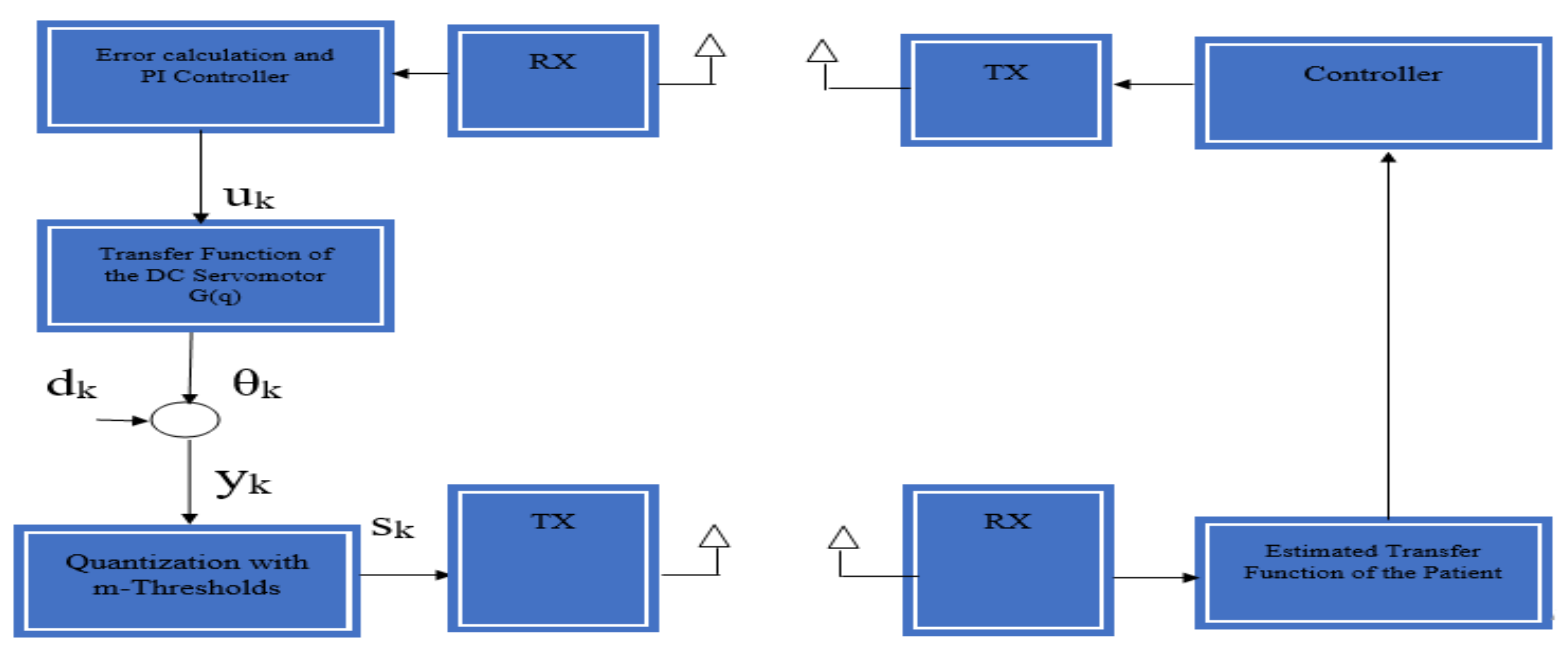

Fig 8: DC Servomotor identification closed loop braking control system

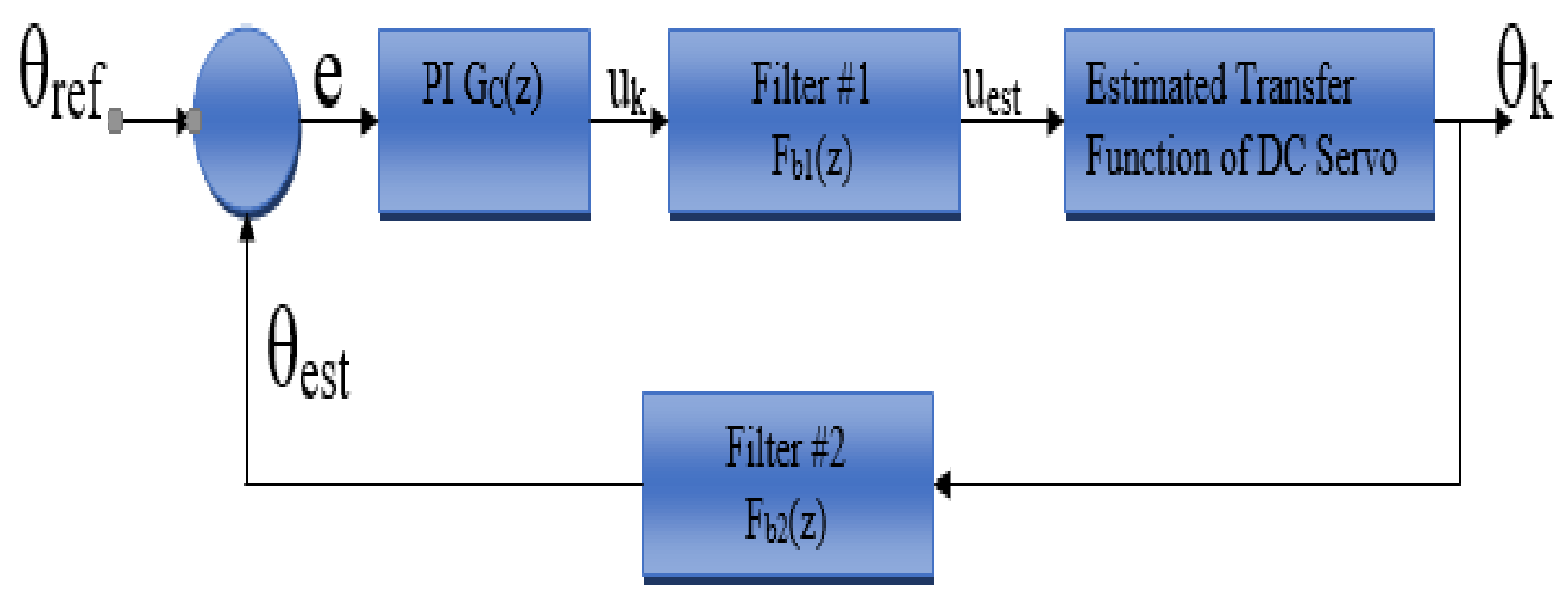

Fig 9: Simplified equivalent DC Servomotor closed loop braking control system

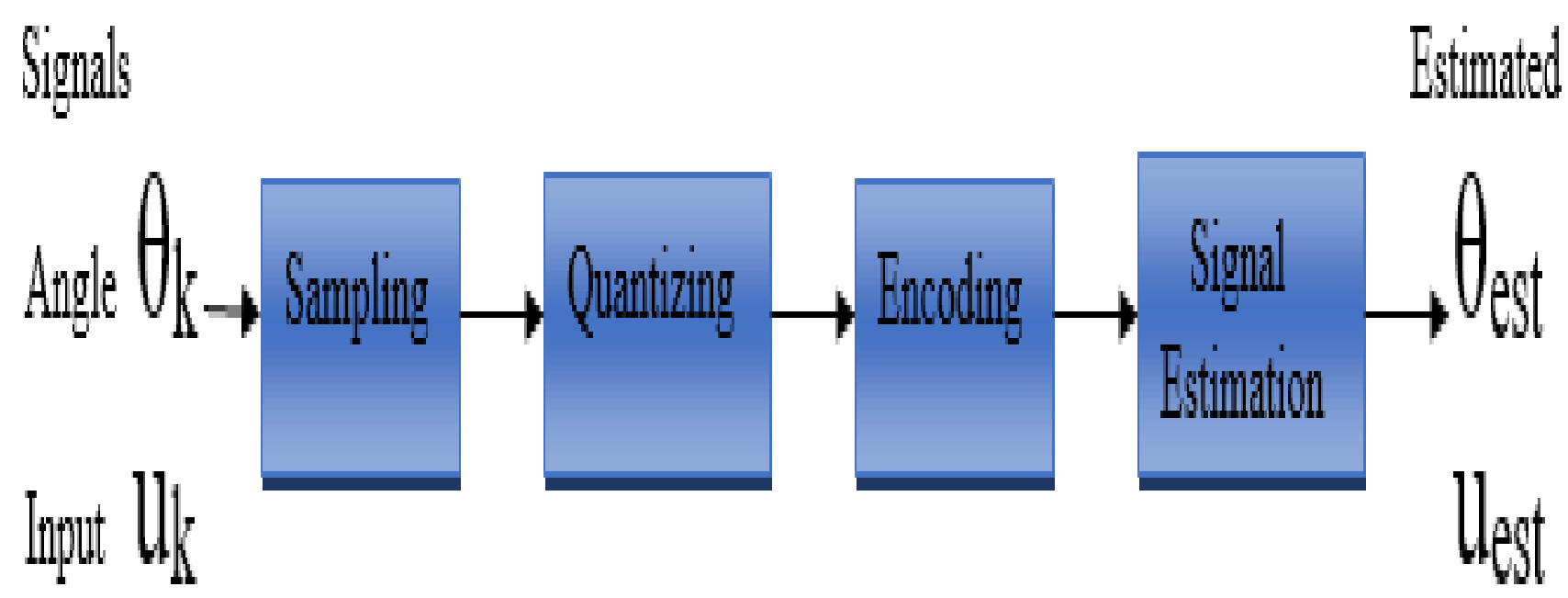

Fig 10: Signal estimation process 


\subsection{The Effect of Signal Estimation on The Performance of Closed Loop System}

Two communication blocks, shown in Figure 3. Analysis of such systems can be carried out using the same methods as in Section 3. Using some typical scenarios to demonstration design variables and their impact, and provide some guidelines

Feedback performance of the response under different values of Signal Averaging step size $\alpha$

The performance of the DC servomotor system using both communication channel blocks 1 and 2 .

\section{Example 2:}

Consider the same DC Servomotor specifications as in Example 1. In this example the sampling intervals for both communication channels $T_{S}=0.001 \mathrm{sec}$, Then the signal estimator (4) is applied. Three cases are considered for $\alpha$. Figure 11 shows the step response of the closed-loop. Figures 12 and 13 expand performance evaluation to the ramp and sinusoid inputs.

Feedback performance of the response under different values of Sampling Intervals $\mathbf{T}_{\mathbf{S}}$.

\section{Example 3:}

Consider the same DC Servomotor specifications as in Example 1 with fixed step size $\alpha=0.60$. Then the signal estimator (4) is applied. The sampling interval $T_{S}$ varied to assess their impacts on feedback performance. Figure 14 shows the step response. Similarly, Figure 15 is for the ramp input, and Figure 16 is for the sinusoidal input.

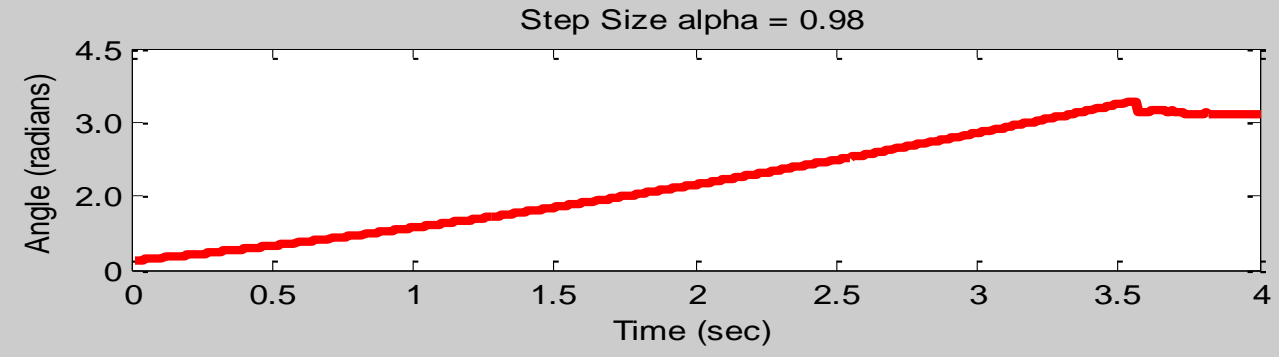

Step Size alpha $=0.9$

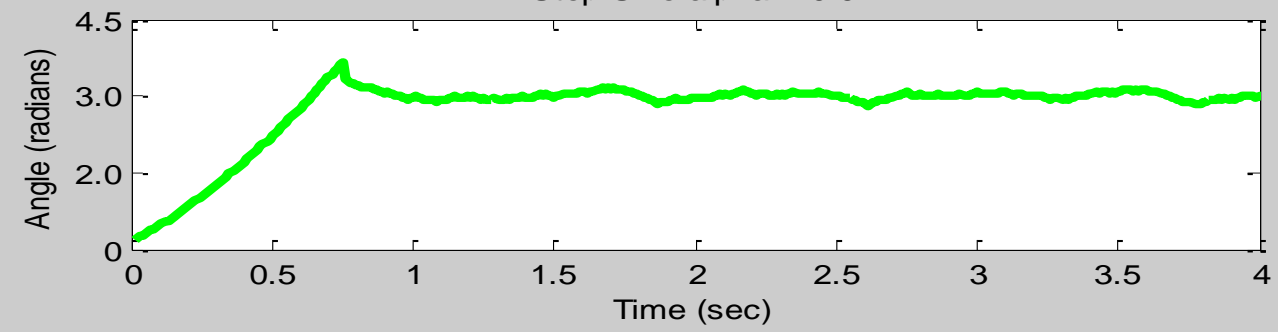

Step Size alpha $=0.6$

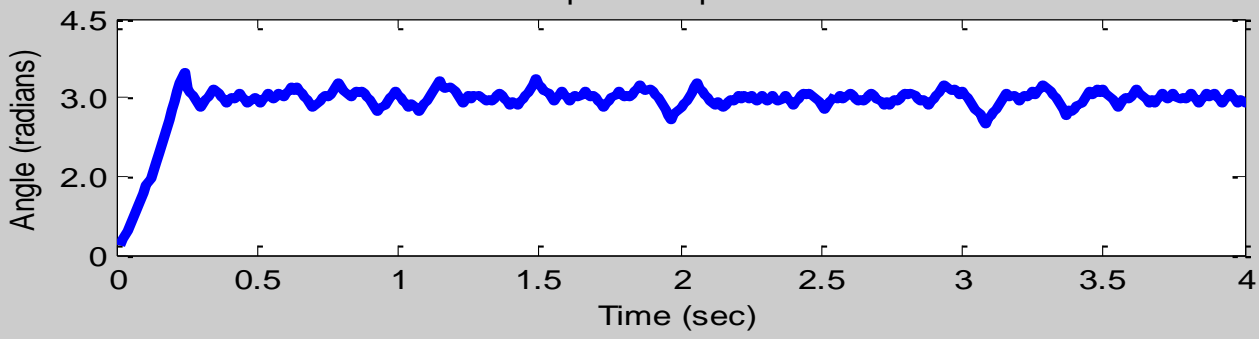

Fig 11: Step response for different step sizes alpha 


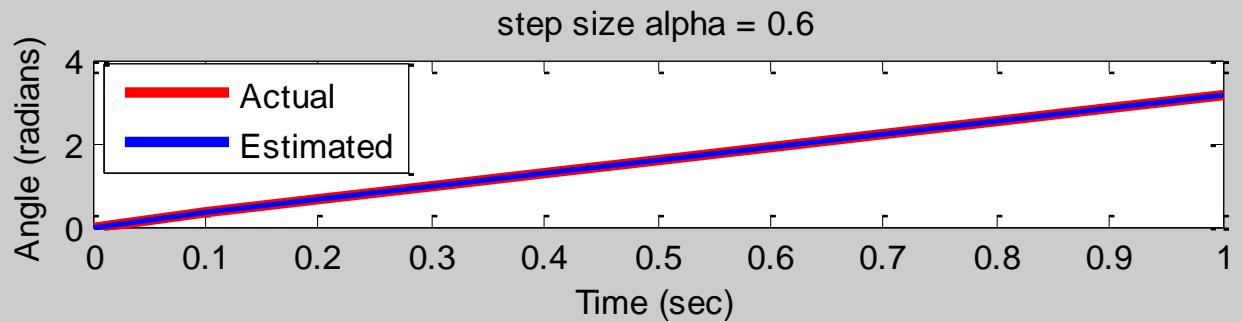

step size alpha $=0.9$

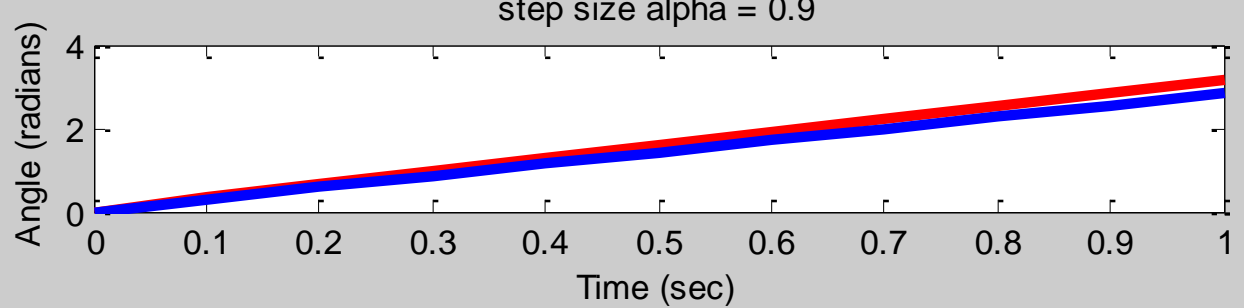

step size alpha $=0.98$

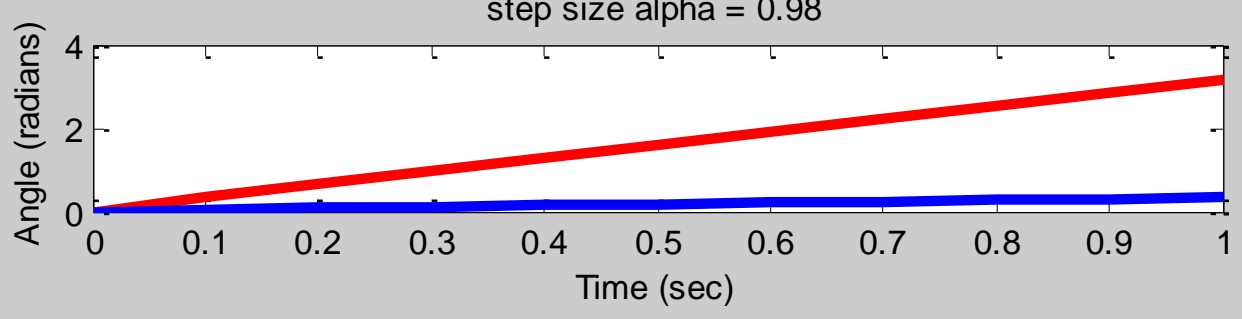

Fig 12: Ramp response for different step sizes alpha

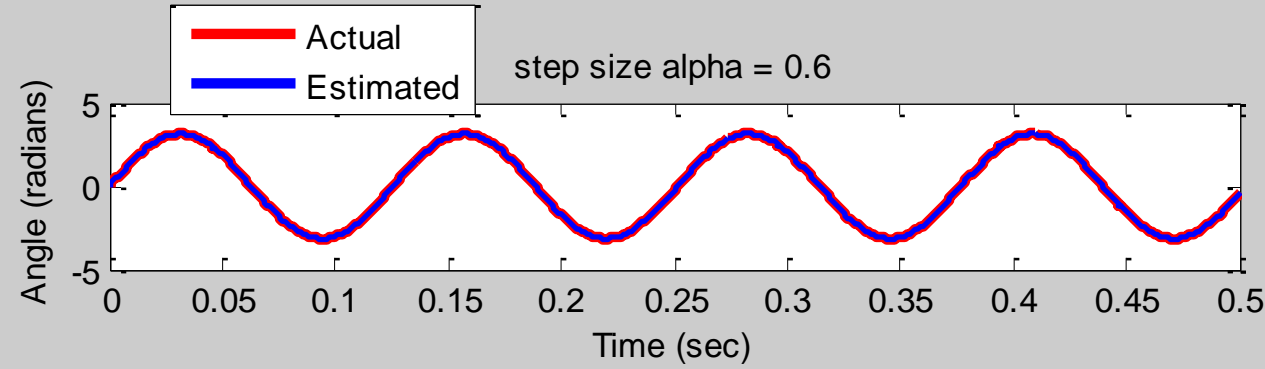
step size alpha $=0.9$
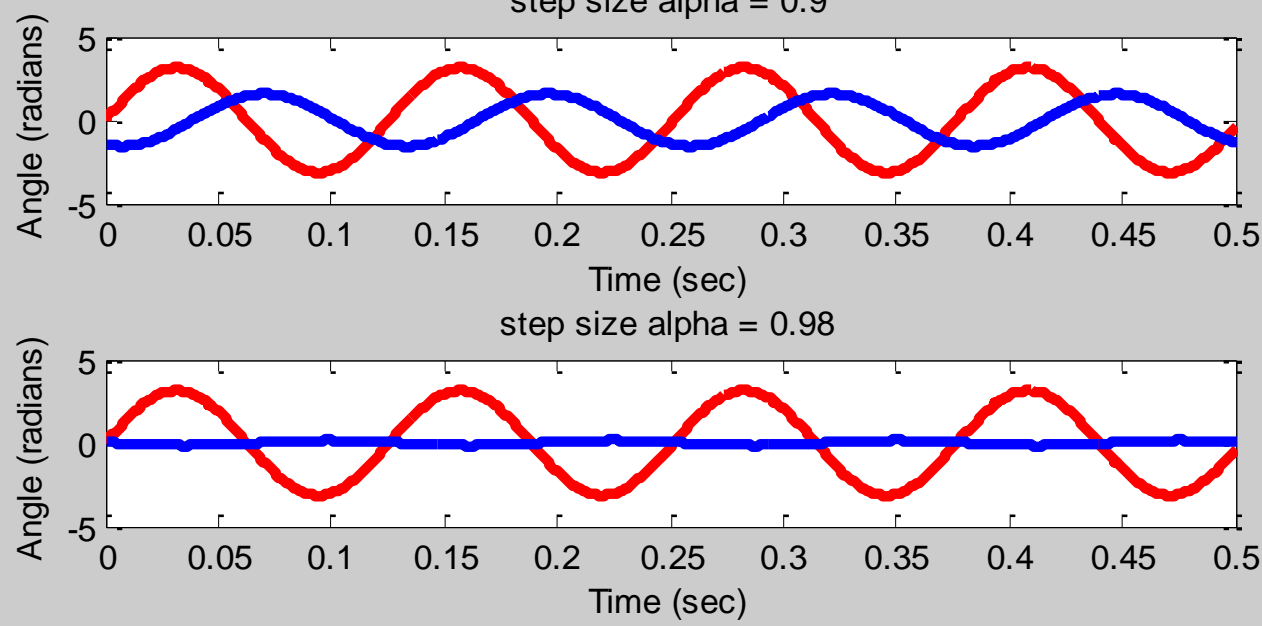

Fig 13: Sinusoidal response for different step sizes alpha 


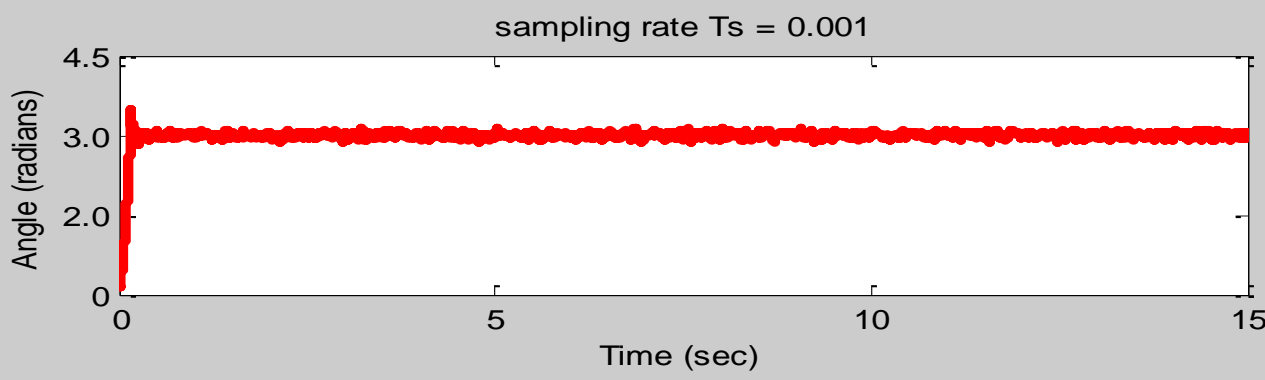

sampling rate $\mathrm{Ts}=0.01$

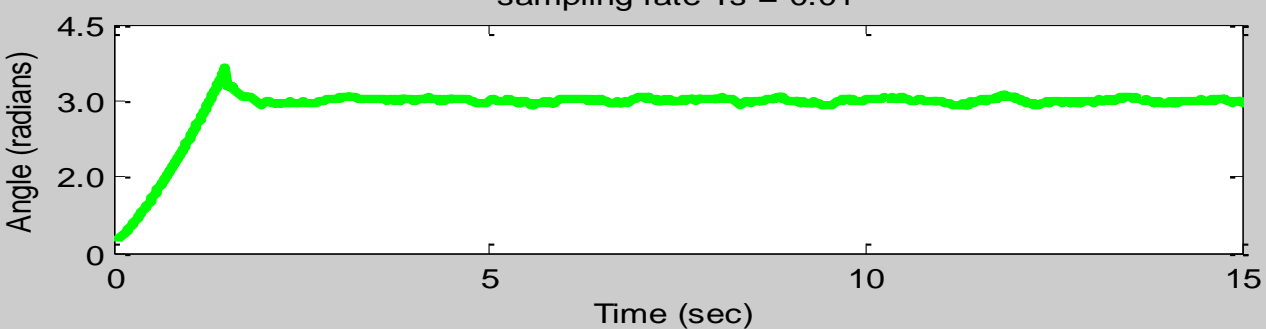

sampling rate $\mathrm{Ts}=0.1$

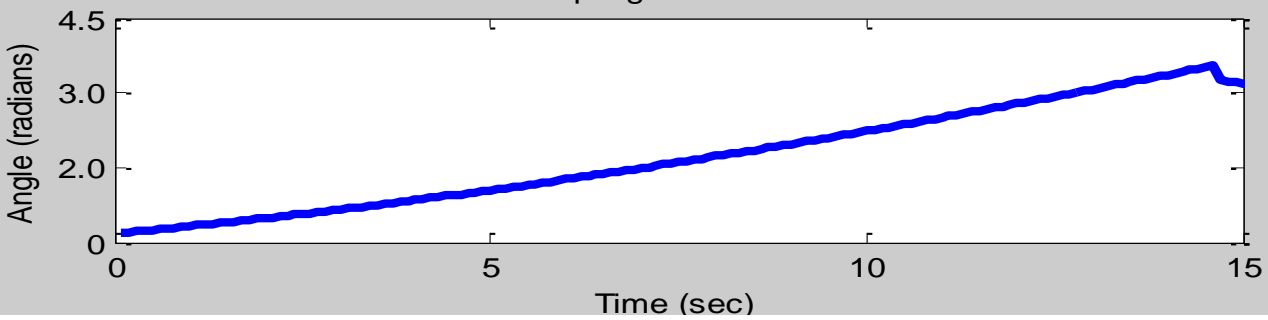

Fig 14: Step response for different sampling time $T_{S}$
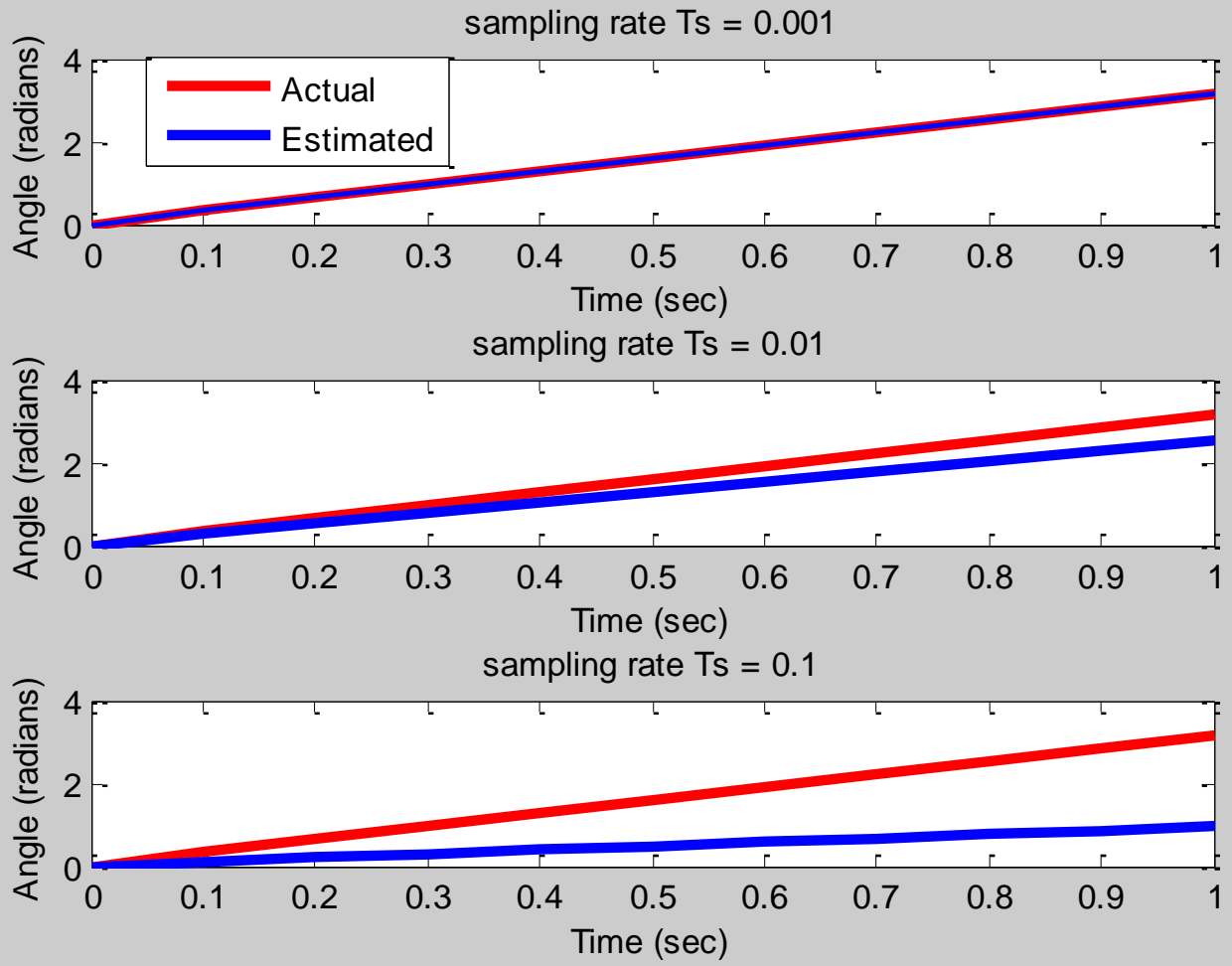

Fig 15: Ramp response for different sampling time $T_{S}$ 


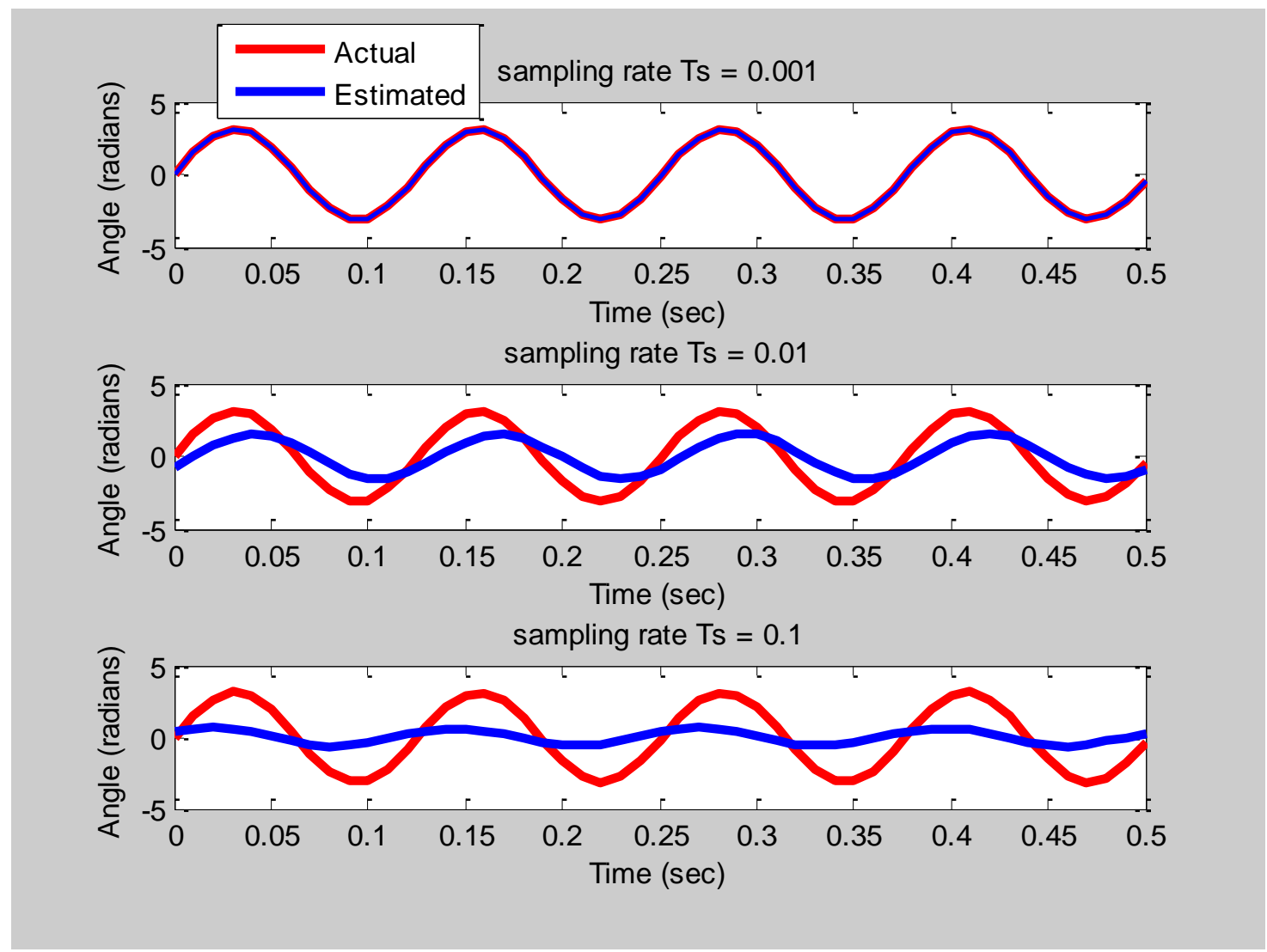

Fig 16: Sinusoidal response for different sampling time $T_{S}$

\section{Discussions:}

From Figure 11, 12, and 13 it can be seen that, if the signal averaging step size $\alpha$ increased, the rise time, settling time, and peak times will increase also. On the other hand, the overshoot becomes smaller. This is because of the data window sizes, such that Larger step size means larger data windows and slower dynamic behavior from the filters. The main implication is that if tracking performance (such as acceleration) is essential, then small $\alpha$ values should be used.

From Figure 14, 15, and 16 as Ts increases, the rise time, settling time, and the peak times increase, while the overshoot reduces. It is cleared that to retain desirable performance, $\alpha$ should be adapted according to sampling times of the system when communication data flow rates fluctuate from communication traffic conditions.

\subsection{Practical Experiment}

\subsection{Experimental Setup}

Using the Arduino and servo motor together to create an intelligent system to modify the traditional brake system in cars and vehicles, which is based on principle brake pedal and convert it into a system switches to be installed on the driver's steering wheel.

A prototype for the DC servomotor system is implemented. The following components used in this experiment are: Arduino uno, servomotor, breadboard, wires, push button, glass tube, plastic arm. In this experiment the following steps are followed:

1) prepare tools, 2) prepare a wooden board to represent the model, 3 ) install the tube and the servomotor in place on the board, 4) install four switches on breadboard Each switch represents a different speed braking, 5) connect the switches and the servo motor to Arduino, 6) attach the plastic arm to the end of the motor, 7) pass the plastic arm inside the tube.

Figure 17 shows the implementation of closed loop DC servomotor using Arduino microcontroller.

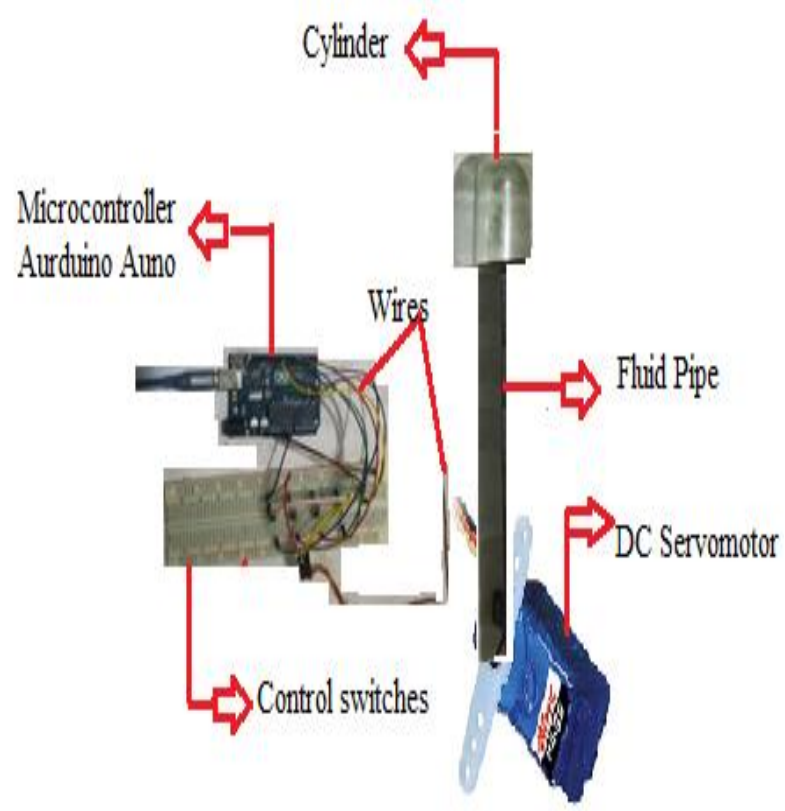

Fig 17: implementation of closed loop DC servomotor using Arduino microcontroller 
Table 1 shows the experimental results of the closed loop circuit shown in figure 17.

Table 1: Experimental results of closed loop DC servomotor

\begin{tabular}{|c|c|c|c|}
\hline Switches & Speed & Angle & Delay \\
\hline 1 & Very high & 60 & 1500 \\
\hline 2 & High & 90 & 1300 \\
\hline 3 & Medium & 120 & 1150 \\
\hline 4 & Low & 180 & 1000 \\
\hline
\end{tabular}

\section{REFERENCES}

[1] J.G. Proakis and M. Salehi, Digital Communications, 5th Edition, McGraw-Hill Higher Education, New York, 2008.

[2] M. Kostadinović, M. Stojčev, Z. Bundalo and D. Bundalo, "Simulation model of DC servo motor control," Proceedings of 14th International Power Electronics and Motion Control Conference EPE-PEMC 2010, Ohrid, 2010, pp. T7-10-T7-14.

[3] M. S. Qureshi, P. Swarnkar and S. Gupta, "Assessment of DC servo motor with sliding mode control approach," 2016 IEEE First International Conference on Control, Measurement and Instrumentation (CMI), Kolkata, 2016, pp. 351-355.

[4] X. Peng, M. Jia, L. He, X. Yu and Y. Lv, "Fuzzy sliding mode control based on longitudinal force estimation for electro-mechanical braking systems using BLDC motor," in CES Transactions on Electrical Machines and Systems, vol. 2, no. 1, pp. 142-151, March 2018.

[5] L. Zhang, L. Yu, Z. Wang, L. Zuo and J. Song, "AllWheel Braking Force Allocation During a Braking-inTurn Maneuver for Vehicles With the Brake-by-Wire System Considering Braking Efficiency and Stability," in IEEE Transactions on Vehicular Technology, vol. 65, no. 6, pp. 4752-4767, June 2016.

[6] T. Soderstom and P. Stoica, System Identification, Printice Hall,1989.

[7] L. Ljung, System Identification: Theory for the User. Prentice-Hall, Englewood Cliffs, NJ, 1987.

[8] L. Y. Wang, J. F. Zhang, and G. Yin, System identification usingbinary sensors, IEEE Transactions on Automatic Control, 48, pp.1892-1907, 2003.
[9] C.E. de Souza, R.M., Palhares, and P.L.D. Peres, Robust H1 filtering design for uncertain linear systems with multiple timevarying state delays, IEEE Trans. Signal Processing, Vol. 49, Issue 3, pp. 569-576, 2001.

[10] Fredrik Gustafsson and Rickard Karlsson, Statistical results for system identification based on quantized observations, Automatica,45(12): 2794-2801, 2009.

[11] K. Ogata, Discrete-Time Control Systems, Prentice-Hall, Inc. Upper Saddle River, NJ, 1987.

[12] L. Y. Wang and G. Yin, Asymptotically efficient parameterestimation using quantized output observations, Automatica, 43, pp. 1178-1191, 2007.

[13] Le Yi Wang, George Yin, Ji-feng Zhang, Yanlong Zhao, System Identification with Quantized Observations, Boston, MA: Birkh"auser, 2010.

[14] Mohammad A Obeidat, Le Yi Wang, Feng Lin, Realtime parameter estimation of PMDC motors using quantized sensors, IEEE Transactions on Vehicular Technology, Vol. 62, Issue 6, pp. 1-10, 2013.

[15] Zhibin Tan, Le Yi Wang, Hong Wang, Signal Averaging for Noise Reduction in Anesthesia Monitoring and Control with Communication Channels, Journal of Biomedical Science and Engineering, Vol. 2-7, pp. 564573, 2009.

[16] T. Wigren, Convergence analysis of recursive identification algorithms based on the nonlinear Wiener model, IEEE Transactions on Automatic Control, Volume 39, Issue 11, pp. 2191 - 2206, 1994.

[17] J. Voros, Parameter identification of Wiener systems with multisegment piecewise-linear nonlinearities, Systems and Control Letters, Volume 56, Issue 2, pp. 99 - 105, 2007.

\section{AUTHOR'S PROFILE}

Mohammad A Obeidat is an assistant professor in power and mechatronics department at Engineering college at Tafila Technical University in Jordan. He received his $\mathrm{PhD}$ in Electrical Engineering, Wayne State University, in 2013 with Excellent GPA. his M.Sc degree in Electrical Engineering, Yarmouk University, Jordan, in 2006, and his B.Sc degree in Electrical Engineering, Jordan University of Science \& Technology, Jordan, in 1999; and He is a member of IEEE, Tau Beta PI Honor Society, Golden Key Honor Society. He was given the honor to be a Sigma Xi member from the Board of Governor, in 2012. His research interest is in Control systems, Mechatronic, signal processing, Fuzzy logic, Identification and estimations and Intelligent systems. 